Microring resonator-based optical router for photonic networks-on-chip

This content has been downloaded from IOPscience. Please scroll down to see the full text. 2016 Quantum Electron. 46655

(http://iopscience.iop.org/1063-7818/46/7/655)

View the table of contents for this issue, or go to the journal homepage for more

Download details:

IP Address: 218.199.141.19

This content was downloaded on 30/08/2016 at 03:46

Please note that terms and conditions apply.

You may also be interested in:

Wafer-Level Testable High-Speed Silicon Microring Modulator Integrated with Grating Couplers Xiao Xi, Zhu Yu, Xu Hai-Hua et al.

All-optical flip-flop and inverter using adjacent lasing wavelengths by semiconductor microring laser

Tominari Miyamoto, Shu Umehara, Hiroki Kobayashi et al.

High-speed silicon-based microring modulators and electro-optical switches integrated with grating couplers

$\overline{X i}$ Xiao, Yingtao Hu, Haihua Xu et al.

A load balancing bufferless deflection router for network-on-chip

Zhou Xiaofeng, Zhu Zhangming and Zhou Duan

Optical interconnection networks for high-performance computing systems

Aleksandr Biberman and Keren Bergman

The Photonic Band Gaps in the Two-Dimensional Plasma Photonic Crystals with Rhombus Lattice Zhang Kaiming and Sun Dongsheng 


\title{
Microring resonator-based optical router for photonic networks-on-chip
}

\author{
Zhihua Yu, Qi Zhang, Xin Jin, Juan Zhao, Hadi Baghsiahi, David R. Selviah
}

\begin{abstract}
We report the design and analysis of a non-blocking microring resonator-based optical switched router, which can be used as a switch node to construct a large photonic routing network on chips. The proposed optical router has sixteen microrings, fourteen crossings and four $90^{\circ}$ waveguide bends, which could be tuned through the thermo-optic (TO) or electro-optic (EO) effect. Compared with a previously described $5 \times 5$ optical switching router, our router comprises fewer microring resonators (MRRs), crossings and bends, which results in a more compact design, a higher switching speed, a lower loss and a lower optical power consumption. In addition, all the rings operate at the same wavelength making it scalable to a network of any size.
\end{abstract}

Keywords: optical router, network-on-chip, microring resonator, man-machine interface, optical switch, photonic networks, silicon photonics, interconnects.

\section{Introduction}

Recently, photonic networks-on-chip (NoCs) have attracted interest of many researchers for such applications as interconnection of many-core distributed, cluster cloud computing systems. As pointed out in the International Technology Roadmap for Semiconductors (ITRS), optical communications on a chip is the only alternative interconnection technology that promises high-data-rate signal transmission and low power consumption [1, 2]. Several network architectures [3], such as mesh, torus, crossbar, fat tree and Clos, have been analysed to construct efficient photonic NoCs [4, 5]. According to the configurability of the routing patterns, they can be divided into two categories. One is a wavelength-selective passive network which has a fixed routing pattern defined by the design of the network. The path between the source and the destination is established through the dynamic selection of a

Zhihua Yu School of Automation, China University of Geosciences (Wuhan), Wuhan, Hubei 430074, China; Department of Electronic and Electrical Engineering, University College London, UCL, London WC1E 7JE, UK; e-mail: yuzhihua@cug.edu.cn;

Qi Zhang, Xin Jin, Juan Zhao School of Automation, China University of Geosciences (Wuhan), Wuhan, Hubei 430074, China; Hadi Baghsiahi, David R. Selviah Department of Electronic and Electrical Engineering, University College London, UCL, London WC1E 7JE, UK

Received 5 November 2015; revision received 31 January 2016 Kvantovaya Elektronika 46 (7) 655-660 (2016)

Submitted in English specific wavelength at the source or the destination [6]. The other is a switched network which dynamically sets a routing pattern through an electronically controlled network. It is claimed that the former can exhibit low latency since the routing network is passive, but the problem is that it requires as many light sources as the network has distinct paths. This leads to problems in scaling due to the necessary physical space and power consumption required by each light source.

In an optical switching network, the design of the optical router is of key importance. In recent years, several researchers and institutes have reported optical on-chip routers [7, 8], with a particularly notable design being a five-port optical router designed by Ruiqiang Ji et al. [9]. They used sixteen microring resonators, each having the same radius, and twelve waveguide crossings, with a footprint of $460 \times 1000 \mu \mathrm{m}$. Dynamic switching was implemented using thermo-optical (TO) tuning. Their design demonstrated bidirectional communication between five inputs and five outputs.

In this paper, we propose a new configuration of a fiveport $5 \times 5$ non-blocking optical switching router that can be used as a switch node to construct a photonic NoC. The optical router has sixteen microrings, fourteen crossings and four $90^{\circ}$ waveguide bends, which can be tuned using the thermo-optic or electro-optic (EO) effects to realise various interconnection patterns. In addition, all the rings operate at the same wavelength making it scalable to a network of any size.

\section{Photonic network architectures and routers}

\subsection{Photonic network architectures}

Various approaches have been proposed to integrate electronic and photonic device layers on a single chip integrated circuit or between chips on a printed circuit board [6] However, it is generally agreed that photonic networks-onchip should be in the form of multilayer electrical-control architecture, with a topology such as a mesh or torus $[10,11]$. Figure 1 shows a universal block diagram of a $4 \times 4$ mesh network connecting sixteen different processing elements. Each processing element communicates with a five-port router through the electronic-optical/optical-electronic interfaces in the optical layer. Since there are no optical buffers in this mesh network, it is a photonic circuit-switched network. In contrast with wavelength-routing networks, the routing path of the data from a source to a destination can be configured dynamically by the electronic circuits. 


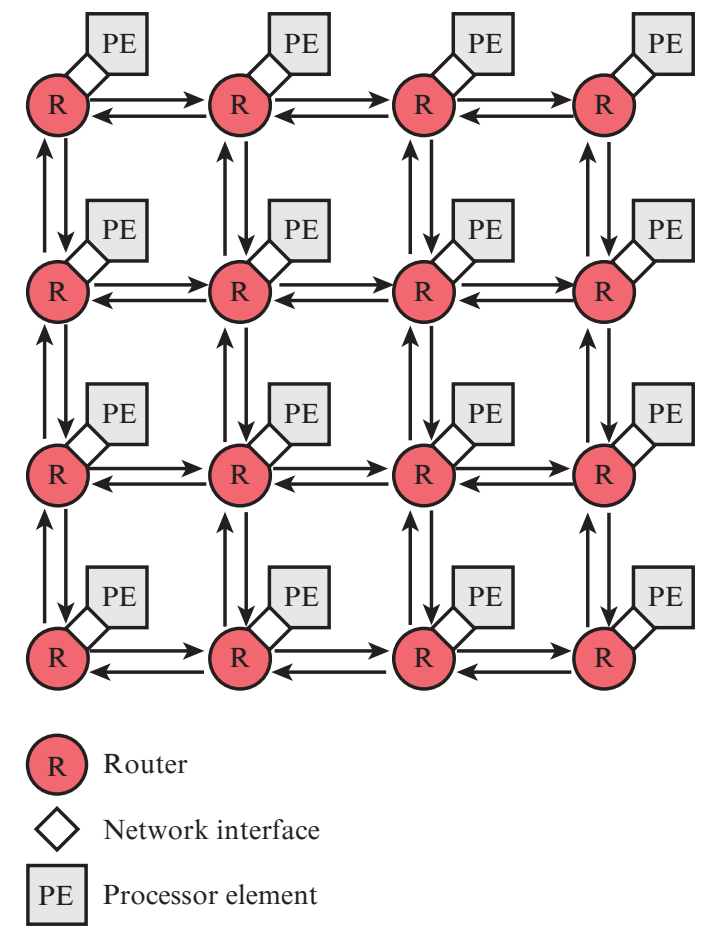

Figure 1. Schematic of a 2D photonic mesh network.

\subsection{Router topology}

Figure 2 shows the five-port optical router with sixteen microring resonators, which is one node of the above photonic mesh network. The configuration includes four $90^{\circ}$ bend waveguides and one straight waveguide to comprise five input ports and five output ports, enabling simultaneous bidirectional communication. Additionally, all of these sixteen mirroring resonators are designed to operate at the same resonance wavelength and are controlled by an electronic circuit integrated on the same chip. Since the MRRs of the router are identical, they have the same structural parameters, which makes their fabrication relatively simple as compared with wavelength selecting networks. Table 1 gives twenty-one pos-

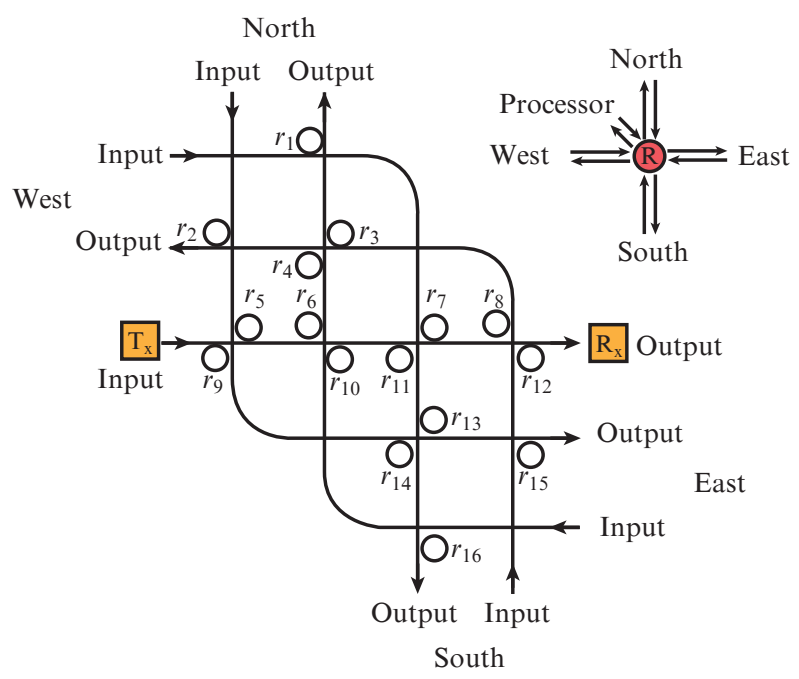

Figure 2. A five-port bidirectional optical router.
Table 1. Optical links of the five-port non-blocking optical router.

\begin{tabular}{llllll}
\hline \multirow{2}{*}{$\begin{array}{l}\text { Input } \\
\text { ports }\end{array}$} & \multicolumn{5}{c}{ Output ports } \\
\cline { 2 - 6 } & East & South & West & North & Output \\
\hline East & - & $r_{16}$ & $r_{4}$ & none & $r_{10}$ \\
South & $r_{15}$ & - & none & $r_{3}$ & $r_{12}$ \\
West & $r_{13}$ & none & - & $r_{1}$ & $r_{7}$ \\
North & none & $r_{14}$ & $r_{2}$ & - & $r_{5}$ \\
Input & $r_{9}$ & $r_{11}$ & $r_{8}$ & $r_{6}$ & none \\
\hline
\end{tabular}

sible optical links between different input and output ports. Any of the four port compass directions is forbidden to connect with itself. Moreover, each of the twenty-one optical links is established by a specific resonant microring with the number $m$ (labelled $r_{m}$ ) or a waveguide directly (labelled as 'none' with the meaning that no microring is required).

Unlike metal track interconnects, optical waveguide crossings can be employed in photonic circuits, and due to this property, complex routing can be completed in a $2 \mathrm{D}$ layer. However, waveguide crossings and bends also bring additional transmission loss, reflection loss and crosstalk. Therefore, the topology of the router should be designed to minimise the number of crossings and bends. As bends have more loss than crossings, it is better to minimise the number of bends at the expense of a slightly increased number of crossings.

The proposed five-port optical router has sixteen MRRs, fourteen crossings and four bends. Compared with other fiveport optical routers proposed in $[3,7,8,10]$, our router comprises fewer crossings and bends and fewer MMRs. Table 2 compares four typical five-port optical routers. Reduction of the number of crossings and bends means lower insertion loss and lower crosstalk, which significantly limits the scalability of photonic NoCs. In addition, the reduction of MRRs can also offer overall benefits in terms of device area and power consumption, although it has no effect on individual optical links using waveguides without MMRs, such as East $\rightarrow$ North, which require no power consumption.

\section{MRR-based switch design}

\subsection{MRR design}

The basic switch element is an add-drop filter comprising a MRR coupled with a waveguide crossing. Figure 3 a shows the schematic of the basic building block. The optical signal excited at the input port propagates along the vertical waveguide. When the signal wavelength does not correspond to the resonance wavelength of the microring resonator, the optical signal continues to propagate without coupling to the other orthogonal (horizontal) waveguide. When the signal wavelength is at the resonance wavelength, the optical signal couples into the microring resonator and couples out to the other waveguide. Figure $3 \mathrm{~b}$ shows the numerically simulated transmission spectra using the transfer matrix method.

In order to transfer more power to the drop waveguide at resonance wavelengths, the transmission spectrum extinction ratio $\mathrm{ER}\left[\mathrm{ER}=-10 \lg \left(P_{\min } / P_{\max }\right)\right]$ is given as a function of $k_{1,2}$ and $A$. According to [12], large $k_{1,2}$ and large $A$ (low transmission loss) give a high ER. Hence, strong coupling and low microring transmission loss would be the best choice for this router, just as Fig. 4 demonstrates the normalised 
Table 2. Comparison of five-port optical routers.

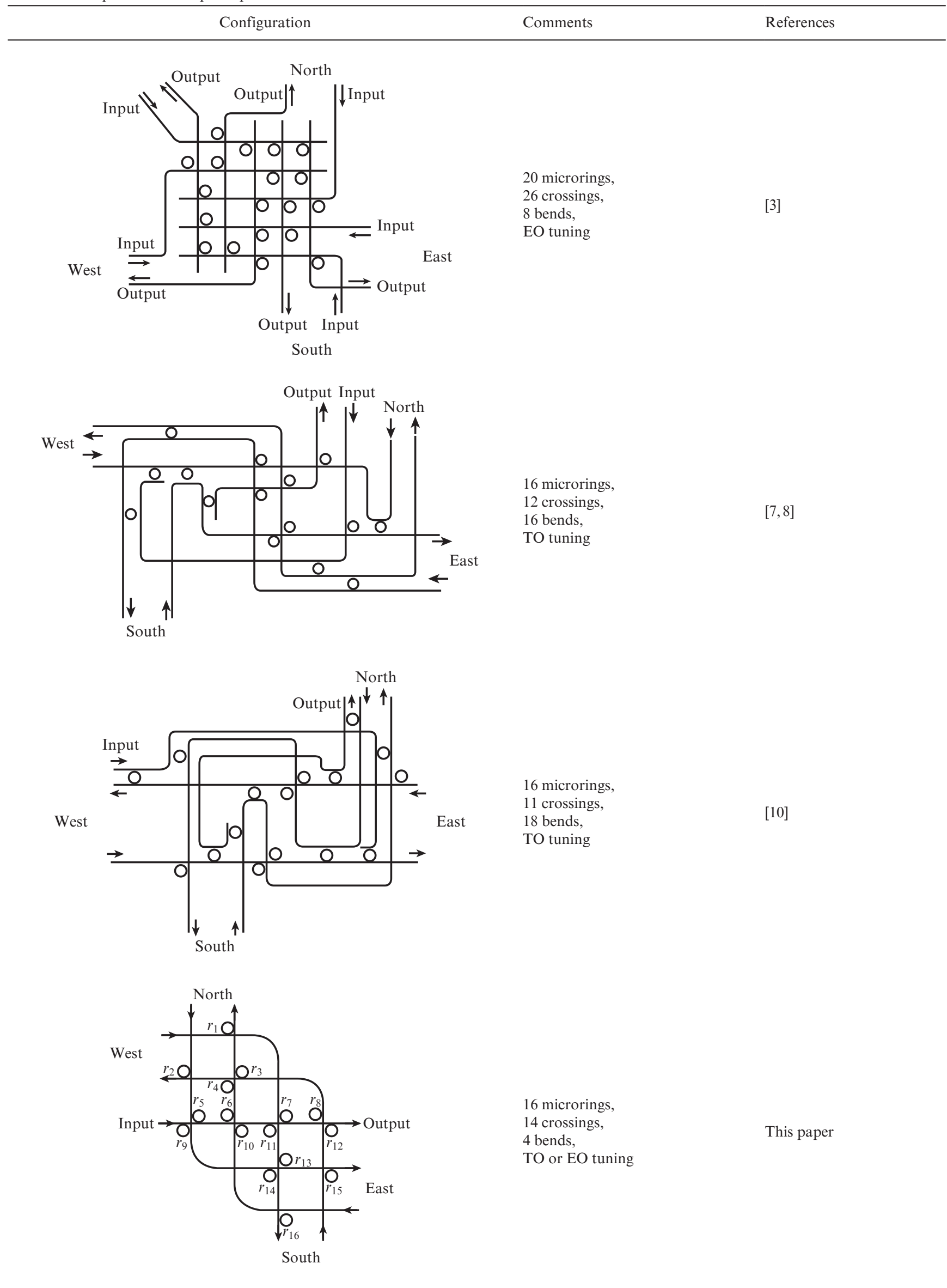



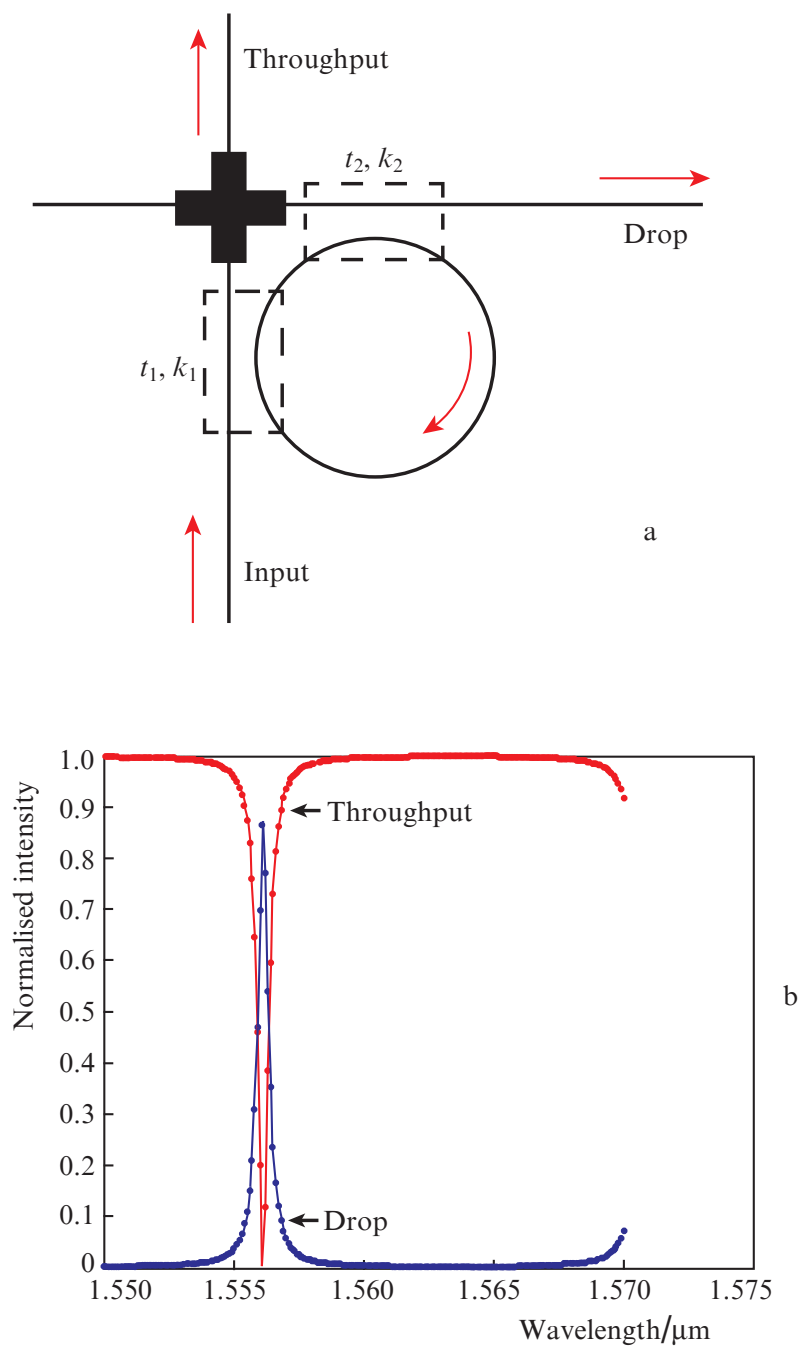

Figure 3. (a) Basic switch element using a microring resonator sidecoupled to a MMI waveguide crossing $\left(k_{1,2}\right.$ and $t_{1,2}$ are the field coupling and transmission coefficients, respectively) and (b) straight through and drop port numerically simulated transmission spectra using the transfer matrix method $\left(k_{1}=k_{2}=0.3, A=0.993\right.$ is the microring round-trip amplitude transmission factor).

intensity of the drop waveguide at resonance wavelengths at different $k_{1,2}$ and $A$.

\subsection{Switch design}

According to the above discussion, when the resonance condition is satisfied, light is passed to the drop port instead of the through port. Thus, by tuning the refractive index of silicon and hence the effective index of the mode, we can switch a single wavelength between the through port and the drop port. The shift of the resonant wavelength can be expressed as $\Delta \lambda=\lambda_{0} \Delta n_{\text {eff }} / n_{\text {eff }}$, where $\Delta n_{\text {eff }}$ is the shift of the microring effective index, and $\lambda_{0}$ is the original resonance wavelength.

There are two main ways to tune the refractive index in silicon. One is to use the TO effect [13, 14]. By heating the microring to change its refractive index, the resonance wavelength of the microring resonator will shift. The TO effect for silicon at $1.5 \mu \mathrm{m}$ can be expressed as:

$$
\frac{\mathrm{d} n_{\text {eff }}}{\mathrm{d} T}=1.86 \times 10^{-4} K^{-1} .
$$

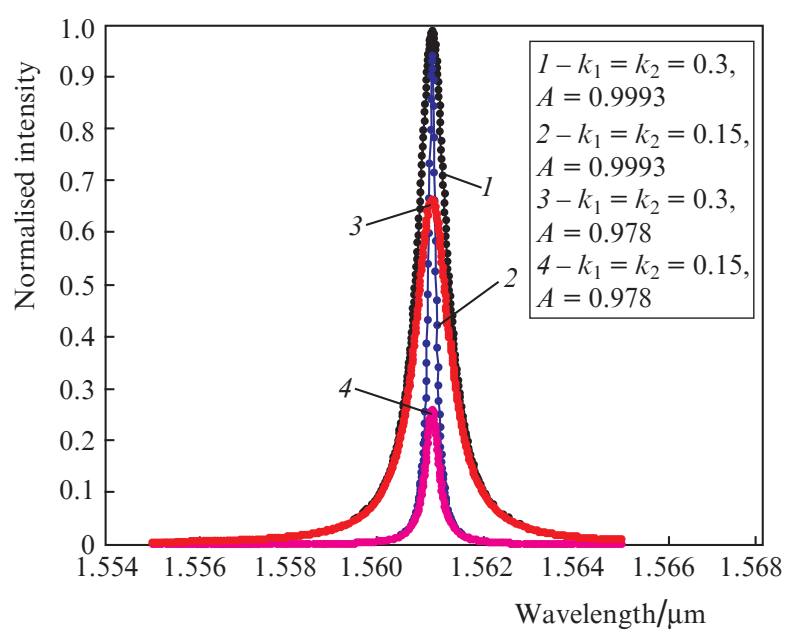

Figure 4. (Colour online) Normalised intensity of the drop waveguide near the resonance wavelength at different $k_{1,2}$ and $A$.
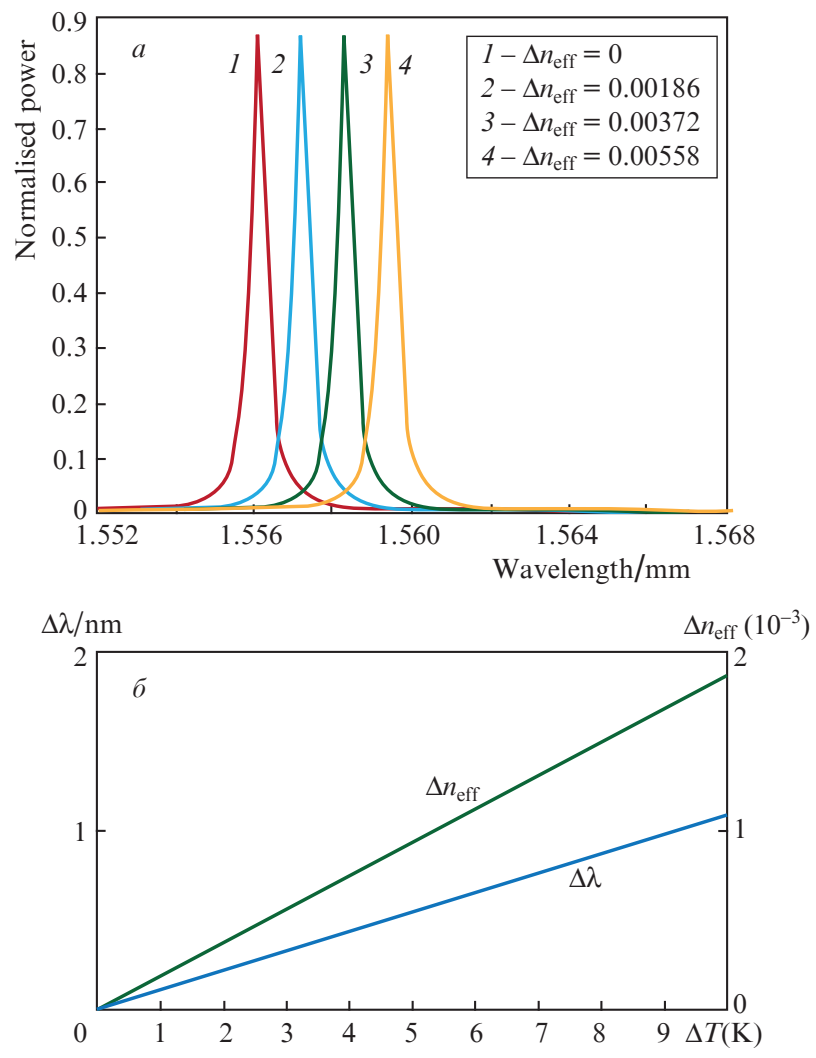

Figure 5. (a) Resonance wavelength shift in the microrings that is caused by the shift in effective index and (b) equivalent temperature shift.

According to (1), we can understand how the temperature $(T)$ relates to the effective index $(n)$ in silicon and in turn shifts the resonant wavelength of the ring. When the silicon microring's temperature rises by $5 \mathrm{~K}$, the effective index will increase by 0.001 , and the resonance wavelength will be redshifted by about $0.5 \mathrm{~nm}$ as shown in Fig. $5 b$.

The other method for wavelength tuning is the EO effect $[15,16]$, known as the free carrier plasma dispersion effect in silicon. The empirical equations describing the changes in the refractive index $\Delta n$ and the accompanied absorption coefficients $\Delta \alpha$ as functions of changes in free electron and hole 
concentrations $\Delta N_{\mathrm{e}}\left(\mathrm{cm}^{-3}\right)$ and $\Delta N_{\mathrm{h}}\left(\mathrm{cm}^{-3}\right)$ at $1550 \mathrm{~nm}$ have the form

$$
\begin{gathered}
\Delta n=\Delta n_{\mathrm{e}}+\Delta n_{\mathrm{h}}=-\left[8.8 \times 10^{-22} \Delta N_{\mathrm{e}}+8.5 \times 10^{-18}\left(\Delta N_{\mathrm{h}}\right)^{0.8}\right], \\
\Delta \alpha=\Delta \alpha_{\mathrm{e}}+\Delta \alpha_{\mathrm{h}}=8.5 \times 10^{-18} \Delta N_{\mathrm{e}}+6.0 \times 10^{-18} \Delta N_{\mathrm{h}} .
\end{gathered}
$$

The advantage of using the EO effect is that the resonance has a faster switching time in silicon, which can be below 100 ps. However, the wavelength shift due to the EO effect is limited to about $\sim 2 \mathrm{~nm}$, while the TO effect has been shown to provide a large wavelength shift on the order of $\sim 20 \mathrm{~nm}$, because the TO effect causes very strong refractive index tuning in silicon $\left(\Delta n \sim 1 \times 10^{-2}\right)$. Thus, in practice, the TO effect is usually used for optical circuit switches [14-16]. However, the electro-optical switching scheme would be useful in the future, because it can offer a more compact design, a higher switching speed and a lower power consumption.

\section{Analysis of optical power consumption}

The optical power loss in this router is mainly due to the offstate through-port transmission loss, the on-state drop-port transmission loss, the waveguide crossing loss and the bend loss, ignoring the input and output coupling losses. Here, the waveguide propagation loss is neglected, as the interconnect distance is microns and the propagation loss can be as low as $\sim 1.7 \mathrm{~dB} \mathrm{~cm}^{-1}$. Since the waveguide bend radius is much larger than the microring's bend radius (see Fig. 3a), the bend loss could be described as the propagation loss, the waveguide
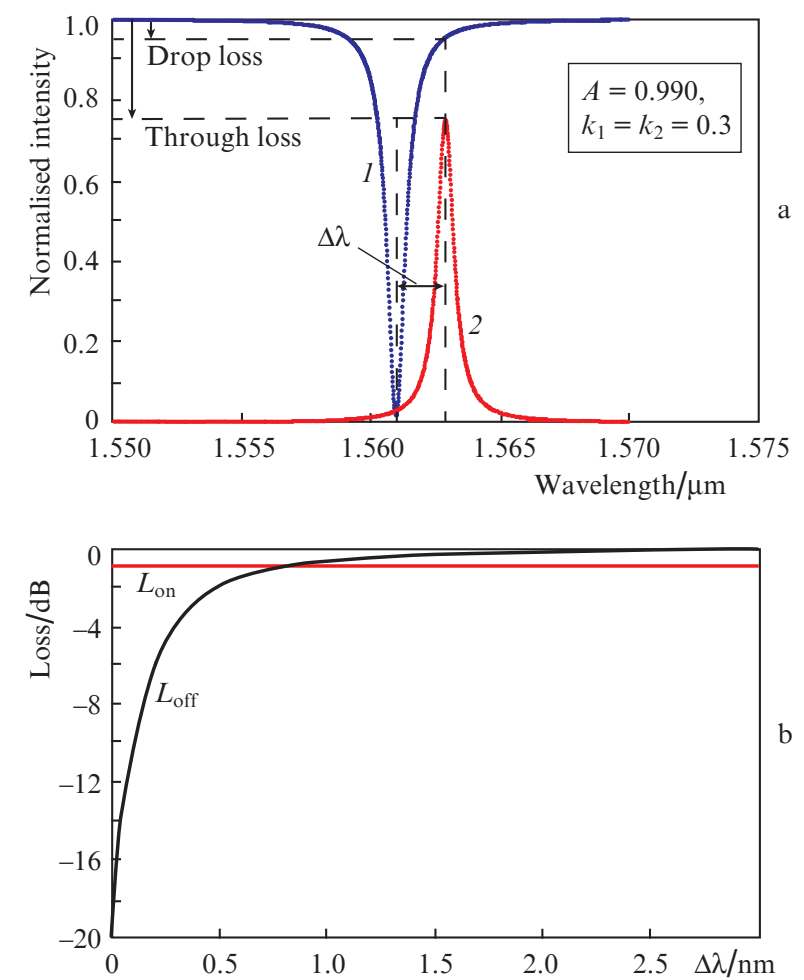

Figure 6. (a) Calculated through- and drop-port transmission spectra at (1) off- and (2) on-states for a single switch element, as well as (b) calculated off-state through loss $\left(L_{\text {off }}\right)$ and on-state drop-port loss $\left(L_{\text {on }}\right)$ with on-state resonance wavelength shifts. bend loss being also neglected. Thus, the optical path loss incurred by the router depends only on the number of the on/off-state switch elements that the light passes and the number of waveguide crossings that the light passes in its final route. As shown in Fig. 6a, we can see that the on-state drop loss is much larger than the off-state through loss at the same wavelength at which the drop loss is maximal. But in a long light path with this router, the number of off-state switch elements may far exceed the number of on-state switch elements. Therefore, comparing with the drop-port loss, the accumulated through loss could be the dominating factor.

Let us consider a $6-\mu \mathrm{m}$-radius microring resonator and use the following calculation parameters: $n_{\mathrm{eff}}=2.65, A=$ $0.990, k_{1}=k_{2}=0.3$ (according to Poon et al. [3]). Then we use the transfer matrix method to calculate the off-state throughport transmission loss and the on-state drop-port transmission loss. As shown in Fig. 6a, the on-state drop-port transmission loss and the off-state through-port transmission loss depend on the wavelength detuning $\Delta \lambda$ from the off-state resonance wavelength.

In a many-core interconnect network, the maximum scalability of port-to-port interconnectivity mainly depends on the optical power loss, especially that of the highest path loss in the router. So, we need analyse the router's link loss. Here, assuming the red-shift $\Delta \lambda$ to be equal to $1.5 \mathrm{~nm}$, then according to Fig. 6b, we find that the off-state throughput-port transmission loss is $L_{\text {off }}=-0.86 \mathrm{~dB}$, the on-state drop-port transmission loss is $L_{\mathrm{on}}=-0.86 \mathrm{~dB}$ and the MMI crossing loss is $L_{\text {cross }}=$ $-0.18 \mathrm{~dB}[10]$. The waveguide propagation loss and input and output coupling losses are neglected. Then, for each light path in the router we calculate the optical power loss (see Table 3 ).

Table 3 shows the loss for all twenty-one optical links in the router. We can see from Table 3 that the largest light loss is in the Inpu-West link, where the optical power loss is $-3.44 \mathrm{~dB}$. This value of the loss includes six off-state throughput transmission losses, four waveguide crossing losses, and one drop-port transmission loss. The smallest light link loss in the router is only $-1.04 \mathrm{~dB}$, comprising only one drop-port transmission loss and one off-state throughput-port transmission loss for the East-South link. Figure 7 shows the transfer spectra of these two extreme links: the largest optical loss link and the smallest optical loss link. In order to reduce the optical link loss in this router (especially the largest link loss), we can shift the wavelength to the red by strengthening the TO or

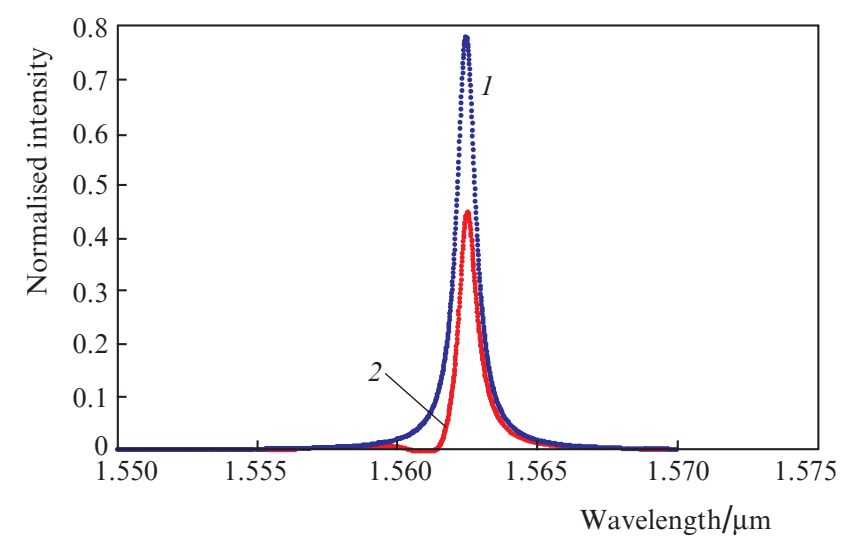

Figure 7. Calculated transmission spectra of optical links for the lowest [East-South, (1)] and highest [Input-West, (2)] losses. 
Table 3. Loss (in $\mathrm{dB}$ ) for twenty-one optical links of the five-port nonblocking optical router (the number of crossings and off-state elements for each path are given in square brackets via a slash).

\begin{tabular}{lccccl}
\hline \multirow{2}{*}{ Input } & \multicolumn{5}{c}{ Output } \\
\cline { 2 - 6 } & East & South & West & North & Output \\
\hline East & - & $-1.04[1 / 0]$ & $-2.48[4 / 3]$ & $-2.28[6 / 4]$ & $-2.48[4 / 3]$ \\
South & $-1.04[1 / 0]$ & - & $-2.28[6 / 4]$ & $-2.66[5 / 3]$ & $-1.52[2 / 1]$ \\
West & $-2.66[5 / 3]$ & $-2.28[6 / 4]$ & - & $-1.34[1 / 1]$ & $-2.18[4 / 2]$ \\
North & $-2.28[6 / 4]$ & $-2.66[5 / 3]$ & $-1.22[2 / 0]$ & - & $-2.96[5 / 4]$ \\
Input & $-2.00[3 / 2]$ & $-2.78[4 / 4]$ & $-3.44[6 / 5]$ & $-2.30[3 / 3]$ & $-1.92[4 / 4]$ \\
\hline
\end{tabular}

EO effect. On the other hand, we can also improve the fabrication process to get larger $k_{1,2}$ and larger $A$ (low transmission loss) to achieve it.

\section{Conclusions}

We have designed and analysed a spatially non-blocking fiveport optical router. The proposed five-port optical router has sixteen MRRs, fourteen crossings and four waveguide bends, which can be tuned through the TO or EO effect. After the detailed analysis of the twenty-one optical links in this router, two ways are proposed to reduce the optical link loss in order to construct large-scale optical interconnection networks on chips.

Acknowledgements. This work was supported by the National Natural Sciences Foundation of China (Grant No. 61205089).

\section{References}

1. Xia F., Sekaric L., Vlasov Y. Nat. Photonics, 1 (1), 65 (2007).

2. Bogaerts W., Heyn P.D., Vaerenbergh T.V., et al. Laser Photonics Rev., 6 (1), 47 (2012).

3. Poon A.W., Luo X., Xu F. Proc. IEEE, 97 (7), 1216 (2009).

4. Yu Z., Han T., Wang G., et al. Optik, 124, 3734 (2013).

5. Shacham A., Bergman K., Carloni L.P. Proc. First Int. Symp. on Networks-on-Chip (NOCS'07) (IEEE Press, 2007) pp 53-64.

6. Pitwon R.C.A., Wang Kai, et al. J. Lightwave Technol., 30 (21), 3316 (2012)

7. Zhou L., Djordjevic S.S. Appl. Phys. A, 95, 1111 (2009).

8. Ji R., Yang L., Zhang L., et al. Opt. Express, 19 (21), 20258 (2011).

9. Ruiqiang Ji, Jiang Xu, Lin Yang. IEEE Photonics Technol. Lett., $25(5), 492$ (2013)

10. Gu H., Mo K., Xu J., et al. Proc. IEEE Computer Society Annual Symp. on VLSI (Tampa, Fl, 2009) pp 19-24.

11. Sherwood-Droz N., Wang H., Chen L. Opt. Express, 16 (20), 15915 (2008).

12. Yariv A. Electron. Lett., 36 (4), 321 (2000).

13. Ji R., Yang L., Zhang L., et al. Opt. Express, 19 (20), 18945 (2011).

14. Magdalena S.N., Tao L., Xuan W. Appl. Phys. Lett., 89, 071110 (2006).

15. Green W.M.J., Hamann H.F., Sekaric L. Proc. Optical Fiber Communication and the National Fiber Optic Engineers Conf. (OFC/NFOEC 2007) (Anaheim, CA, 2007) pp 1-3.

16. Rashed A.M., Papakonstantinou I., Selviah D.R. Proc. IEEE LEOS Annual Meeting Conf. (Rio Grande, Puerto Rico, 2004) pp 457,458 . 